\title{
Supracervical Lymph Node Biopsy under Local Anesthesia: A Cautionary Tale!
}

\author{
Steven D. Boggs ${ }^{1}$, Elizabeth A. M. Frost ${ }^{2}$ \\ ${ }^{1}$ James J. Peters Veterans Administration Hospital, New York, USA \\ ${ }^{2}$ Icahn Medical Center at Mount Sinai, New York, USA \\ Email: elzfrost@aol.com
}

Received 23 February 2015; accepted 23 March 2015; published 25 March 2015

Copyright (C) 2015 by authors and Scientific Research Publishing Inc.

This work is licensed under the Creative Commons Attribution International License (CC BY).

http://creativecommons.org/licenses/by/4.0/

c) (i) Open Access

\section{Abstract}

A middle aged woman was scheduled for supracervical lymph node biopsy under local anesthesia. A scheduling conflict caused a significant operating room delay and she became very nervous. A surgical resident asked for anesthetic assistance in calming the patient. Assured that the case was under local anesthesia only, the anesthesiologist gave the patient soda to drink. In the operating room, the lady could not tolerate the procedure but as she now was at risk for aspiration, the anesthesiologist suggested the case be terminated and rescheduled. The surgeon disagreed and continued but was confronted with substantial bleeding. Emergency induction of general anesthesia was required. Postoperatively bleeding continued requiring re-exploration and intensive care unit admission. The patient developed a compressive left brachial plexopathy. The anatomy of the area indicated that general anesthesia was the preferred technique. The importance of team work and communication is underscored. Complications are more frequent when perioperative changes are made.

\section{Keywords}

Patient Safety, Preanesthetic Assessment, Patient Scheduling

\section{Introduction}

All too often in a busy practice, a surgeon's preference may be to operate using only local anesthesia, believing that time might thereby be shortened. As an anesthesiologist reviews the operation room schedule, he/she will see that the case has been booked for local anesthesia only, thus implying that his/her services are not required to provide general anesthesia or even mild to moderate sedation. The patient is therefore not adequately assessed as to history and physical condition and a plan drawn up for anesthetic and/or analgetic management all essential parts of the preanesthetic examination. Thus should complications arise necessitating a change in management 
patient safety may well be compromised. We present a case that, in hindsight, required more careful evaluation and alternative management from the beginning.

\section{Case}

The patient, a 48-year-old female nurse presented for left supraclavicular lymph node biopsy under local anesthesia. CT scan revealed mediastinal, cervical and supraclavicular lymphadenopathy suspicious for B-cell lymphoma. Additional pertinent history included Hashimoto's thyroiditis treated with levothyroxine, smoking, (1/2 pack per days for many years) and an untreated anxiety disorder.

Following a motor vehicular accident three years previously, a lumbar spinal cord stimulator was placed with some relief of chronic pain. She reported no allergies

She was 67 inches in height and weighed 190 pounds. No other lab values were available.

The surgeon scheduled the patient for open supracervical lymph node biopsy under local anesthesia. The preceding case in the operating room had been delayed significantly and instead of the assigned time of 12:00, the patient was still in the waiting room at 16:30. She was anxious and distressed and the senior surgical resident asked for advice and some guidance. She was sitting on the floor in a changing room, rocking back and forth, saying that she was concerned that the procedure would be cancelled. Her biggest complaint was dry mouth. After verifying with the surgical resident that the procedure was indeed to be accomplished under local anesthesia, the anesthesiologist offered her a diet drink. She accepted, moved to a chair and seemed relieved. The anesthesiologist assured her that all would be done to expedite her case since the preceding case had now finished.

She was taken into the operating room within a few minutes. Approximately 30 minutes later the head nurse called the anesthesiologist to the OR claiming that "The patient is out of control and cannot tolerate the procedure under local anesthesia.” The case had been halted. The anesthesiologist explained that the patient was no longer fasted, she had not been interviewed for an appropriate preanesthetic assessment and if the surgical team was not able to accomplish the procedure under local anesthesia, the case should be aborted and rescheduled with anesthesia assistance at a later date.

Some 5 minutes later, the anesthesiologist was again summoned to the operating room and asked to view the surgical site where a large pool of blood appeared in the supracervical surgical field. Assessing the situation, the patient had no intravenous cannula, the blood pressure cuff was on the right forearm and the saturation monitor was also on the right arm. No EKG leads had been placed.

The patient, who was a nurse and thus had a good understanding of the situation became extremely anxious and, asked "Am I going to die?”. The anesthesiologist reassured her again, verified that she had no allergies and delegated one of the nurses to cannulate a vein while he prepared for a general anesthetic induction. Following pre-oxygenation, a rapid sequence intravenous induction was performed and the patient's trachea was intubated easily.

The surgeons explored the wound more aggressively, but no particular bleeding source was found. An ENT surgeon was consulted and after evaluation he also could find no specific site for bleeding. The wound was closed without difficulty and the patient's trachea extubated without coughing. She was transferred to the stretcher. The surgical resident and anesthesiologist remained with the nurse to observe the patient in the operating room.

Prior to transport to the recovery room, the surgical resident noted that the surgical site had a large hematoma and indicated re-exploration was necessary. The team was called back and a plan for a new procedure formulated. An 18 gauge catheter was placed in the right arm, a blood specimen sent to the blood bank for type and crossmatch and a rapid sequence intubation performed. In addition, the ICU was notified that the patient would require a bed for observation for the night.

Again, the wound was explored but no bleeding source was identified. However, the chief of ENT suggested that the cause was likely bleeding from the superficial or deep cervical veins and that the patient's head should be elevated overnight and a compression dressing applied. The wound was again closed, the patient awakened without difficulty and transported to the ICU where the preceding instructions were observed.

The patient was discharged uneventfully from the facility the next day. However, she developed a compressive left brachial plexopathy due to the hematoma following surgery. Because of her spinal cord stimulator, she did not have a diagnostic MRI. Presently, she has pain in her left axilla, left upper chest and supraclavicular fossa, with left arm strength $4 / 5$, and absent reflexes in her left arm. A review of the surgical specimen revealed fibrous tissue with no lymphoid tissue in the sample. 


\section{Discussion}

This case raises several questions. First, what are the indications for scheduling a case such as this under local anesthesia versus with anesthetic assistance? Second, does the anatomy of the supracervical region offer any suggestions as to choice of the safest management course? Third, what complications might be expected from surgery in this region?

This patient was an educated health care provider who started at a high level of anxiety, in part due to the time delay, which rapidly became compounded by operating room stress, especially as she had been denied sedation. She had several co-morbidities that required more careful evaluation.

Anatomy of the supracervical region of the neck is complicated, packed as the area is with nerve, arterial and venous structures in compact planes between muscle layers [1]. The nodes in this region lie under the platysma muscle, so by definition, a muscle layer must be dissected to achieve a node biopsy [2]. Subsequent to the determination of a neck mass highly suggestive of a lymphoma, it is appropriate to bypass fine needle aspiration (FNA) and go directly to an open node biopsy [3]. While the FNA is less invasive, the open node biopsy should provide abundant material for diagnosis. It also allows the pathologist to interpret both histologic architecture as well as individual cell cytological detail. Oncologists and hematopathologists maintain that open biopsy does not compromise the diagnosis. Seventy five percent (75\%) of lymphomas presenting in the neck are nodal-with classical Hodgkin's lymphoma presenting as painless cervical or supracervical lymphadenopathy [4].

Anesthesiologists and surgeons view the triangles of the neck differently. Anesthesiologists focus on venous access, the cricothyroid membrane and sites for blocks of the brachial plexus. In contrast, depending on the surgical objective, the surgeon may be more focused on superficial anatomy and its relation to facial planes.

There are two major triangles in the neck, anterior and posterior. The anterior triangle is bounded superiorly by the inferior border of the mandible, laterally by the medial border of the sternocleidomastoid and medially by an imaginary midline sagittal line [5]. The anterior triangle is further subdivided into 4 smaller triangles named muscular, submental, submandibular and carotid. However, our focus was on the posterior triangle, which is bounded anteriorly by the posterior border of the sternocleidomastoid muscle, inferiorly by the middle third of the clavicle and posteriorly by the anterior border of the trapezius muscle [6].

A scalene lymph node biopsy is the removal of lymph nodes from an area within the posterior triangle [7]. This region is bounded by the omohyoid muscle, the subclavian and internal jugular veins and lies deep to the omohyoid fascia with the nodes on the anterior scalene muscle. Supraclavicular lymph node biopsies require dissections in the same region but are more superficial.

In a classic paper, Skinner classified the complications of scalene lymph node biopsy into two groups, major and minor [7]. Major complications included pneumothorax, phrenic nerve injury, axillary nerve injury, temporary vocal cord paralysis, myocardial infarction, adult respiratory distress syndrome, and (on the left side) chlyothorax and lymph fistula (4.8\%). Minor complication included wound seroma, temporary Horner's syndromes and supraclavicular nerve injury, in addition to hemorrhage and air embolus (4.3\%). No record of anesthesia involvement is made in these cases. Subsequent study has shown that while the risk of nerve damage is low, it remains finite, on the order of $1 \%$ [8].

To determine if cases similar to ours have been reported, the Anesthesia Closed Claims Project, the Anesthesia Quality Institute (AQI) and the American Association for the Accreditation of Ambulatory Surgery (AAAS) were contacted [9]-[11]. There were no comparable cases in the closed claims file. However, an anesthesiologist might not be named in a malpractice action in a case such as this where he or she came in to aid the patient and surgeon. The AQI is interested in all quality outcomes, including the number of cases in which local anesthetics are converted into general anesthesia. However, at present, no data were available. Finally, the AAAS was not aware of any similar cases in its repository.

As anesthesiologists, we are required to follow monitoring standards during procedures [12]. There are no monitoring standards for surgeons performing procedures under topical or local anesthesia. When moderate sedation (MS) is used, standards again are typically followed, since most practice settings desire accreditation by an agency such as Center for Medicare and Medicaid Services (CMS) [13] or the Joint Commission (JC).

In an anatomically complex surgical area such as the posterior triangle of the neck, ideal operating conditions are necessary if injury to surrounding vascular and nerve structures is to be avoided. Historically, some general surgeons have been trained to always post procedures in the posterior triangle with anesthetic assistance (Prof George Dietrick, personal communication) Studies continue to document that nerve injury still occurs during cervical lymph node biopsies, in 3/363 cases reported by some authors [14]. However, dictums such as this fade 
with the pressures of costs and scheduling.

\section{Lessons Learned}

One only need review the images from an issue of the American Journal of Neuroradiology to realize the vascularity of the posterior triangle [14]. The case emphasizes the need again for close communication between all members of the operating team. It is also important to involve the patient and to determine what level of sedation he/she may require based on individual ability to tolerate the situation. For patient comfort and safety, surgical exposure may be enhanced if the anesthesia team is consulted. When involved, the anesthesia team can ensure that the patient remains comfortable and immobile as necessary and the surgical field is optimally visualized, thus minimizing the risk of damage to surrounding structures.

\section{Conflict of Interest}

The authors have no conflicts of interest to report.

\section{References}

[1] O’Rahilly, M., Carpenter and Swenson (2015) Basic Human Anatomy: A Regional Study of Human Structure. http://www.dartmouth.edu/ humananatomy/part_8/chapter_50.html

[2] Sinnatamby, C.S. (2011) Last’s Anatomy. Elsevier, Amsterdam, 329-454.

[3] Lin, D.T. and Deschler, D.G. (2012) Neck Masses. In: Lalwani, A.K., Ed., Current Diagnosis \& Treatment in Otolaryngology—Head \& Neck Surgery, The McGraw-Hill Companies, New York.

[4] Mandpe, A.H. (2012) Neck Neoplasms \& Neck Dissection. In: Lalwani, A.K., Ed., Current Diagnosis \& Treatment in Otolaryngology—Head \& Neck Surgery, The McGraw-Hill Companies, New York.

[5] http://teachmeanatomy.info/neck/areas/anterior-triangle/

[6] http://teachmeanatomy.info/neck/areas/posterior-triangle/

[7] Skinner, D.B. (1963) Scalene-Lymph-Node Biopsy. New England Journal of Medicine, 268, 1324-1329. http://dx.doi.org/10.1056/NEJM196306132682402

[8] Ferencsik, M., Plukovics, K., Borbenyi, Z., et al. (1990) Peripheral Nerve Injuries as a Rare Complication of Cervical Lymph Node Excision for Diagnostic Purposes. Orvosi Hetilap, 131, 1465-1467.

[9] Domino, K. (2014) American Society of Anesthesiologists Closed Claims File. Personal Communication.

[10] Dutton, R. (2014) Anesthesia Quality Institute. Personal Communication.

[11] Villa, H. (2014) American Association for the Accreditation of Ambulatory Surgery Facilities. Personal Communication.

[12] ASA (2011) Standards for Basic Anesthetic Monitoring. http://www.asahq.org/ /media/Sites/ASAHQ/Files/Public/Resources/standards-guidelines/standards-for-basic-anesthet ic-monitoring.pdf

[13] CMS Hospital CoP (2013) Anesthesia Guidelines. http://www.ahcmedia.com/images/T13143_ColorHandouts.pdf

[14] San Millan Ruiz, D.G.P., Rufenacht, D.A., Delavelle, J., et al. (2002) The Craniocervical Venous System in Relation to Cerebral Venous Drainage. American Journal of Neuroradiology, 23, 1500-1508. 